\title{
State of Charge estimation of lithium-ion power battery based on online parameter identification method and BP neural network
}

\author{
Shuai Qin ${ }^{1, *}$, Dongchen Qin ${ }^{1}$, Hongxia Wu ${ }^{1, *}$, Tingting Wang ${ }^{1}$, Jiangyi Chen $^{1}$, Peizhuo Wang ${ }^{1}$ \\ ${ }^{1}$ School of Mechanical and Power Engineering, Zhengzhou University, Zhengzhou 450001, China \\ *E-mail: 18738172859@163.com, wu_hx@zzu.edu.cn
}

Received: 18 September 2021 / Accepted: 18 October 2021 / Published: 6 December 2021

\begin{abstract}
The accurate estimation of power battery state of charge (SOC) is of great significance to vehicle driving safety and energy management. In this paper, the second-order RC equivalent circuit model is used, the recursive least square method with forgetting factor (FFRLS) is used for online parameter identification, and the extended Kalman filter (EKF) is used for SOC estimation. The off-line data charging and discharging under each working condition of the battery are used as training data, the BP neural network is used for training, and the estimation error of the extended Kalman filter is used as the training output of the neural network to compensate for the error of the extended Kalman filter. FFRLS and EKF are used to ensure the real-time performance of the system, and FFRLS and BP neural networks are used to improve the robustness of the system. It is proven through simulation that this method can effectively improve the accuracy of SOC estimation.
\end{abstract}

Keywords: Online parameter identification; recursive least squares method; BP neural network; extended Kalman filter; robustness; real-time.

\section{FULL TEXT}

(C) 2022 The Authors. Published by ESG (www.electrochemsci.org). This article is an open access article distributed under the terms and conditions of the Creative Commons Attribution license (http://creativecommons.org/licenses/by/4.0/). 